\title{
Protée
}

\section{Ultima Verba ou les silences du tropisme}

\section{Marie Auclair}

Volume 28, numéro 2, 2000

\section{Le silence}

URI : https://id.erudit.org/iderudit/030596ar

DOI : https://doi.org/10.7202/030596ar

Aller au sommaire du numéro

Éditeur(s)

Département des arts et lettres - Université du Québec à Chicoutimi

ISSN

0300-3523 (imprimé)

1708-2307 (numérique)

Découvrir la revue

Citer cet article

Auclair, M. (2000). Ultima Verba ou les silences du tropisme. Protée, 28(2), 79-90. https://doi.org/10.7202/030596ar

\section{Résumé de l'article}

Dans l'oeuvre de Nathalie Sarraute, entre autres dans Ouvrez, le silence est le substrat même du drame que constitue le tropisme, cette sensation infinitésimale servant de matrice à la course du signifiant. Le présent article s'attarde à cette dialectique du silence et de la forme, en tant que le silence se fait signifiant princeps d'une scène de représentation où se joue l'avènement du mot comme rature du silence. Celui-ci ordonne ainsi la monstration du verbe comme performance, comme épreuve du dire, de l'énonciation, constituant ainsi le point de butée mais aussi le point de relance du système signifiant. 


\section{ULTIMA VERBA OU LES SILEN CES DU TRO PISM E}

MARIE AUCLAIR

Il y avait un grand vide sous cette chaleur, un silence, tout semblait en suspens; on entendait seulement, agressif, strident, le grincement d'une chaise traînée sur le carreau, le claquement d'une porte. C'était dans cette chaleur, dans ce silence - un froid soudain, un déchirement. Et elle restait sans bouger sur le bord de son lit, occupant le plus petit espace possible, tendue, comme attendant que quelque chose éclate, s'abatte sur elle dans ce silence menaçant. ${ }^{1}$

Parle-t-on, écrit-on, vit-on pour faire contrecoup à cette implosion de la chaleur du silence? Si la parole perdue est toujours envisagée comme faculté potentiellement récupérable, même artificiellement, ne subit-on pas la perte du silence dans le regret d'avoir, un jour, commis la faute de prendre la parole? Car la parole explose, elle se prend, elle, après avoir été donnée par l'autre pour rompre sa solitude; le silence, lui, se désire, s'évoque, entre autres dans le geste et le mouvement qui le portent au-dehors comme pour en rendre compte, en signer le lieu. Il est le substrat formel d'un impossible discours: celui d'une solitude dialoguant avec elle-même.

Ouvrez aura été la dernière œuvre publiée par Nathalie Sarraute, deux ans avant sa mort. Son point d'arrêt, non pas: Ouvrez en appelle à Tropismes, à L'Ére $d u$ soupçon, à Enfance et peut-être encore plus au théâtre. Ultime dialogue de mots de ce cycle perpétuel que constitue l'écriture sarrautienne, Ouvrez, c'est pour moi du théâtre, si le théâtre constitue «la représentation directe et non médiatisée du discours des personnages" ${ }^{2}$; plus précisément, c'est de la théâtralité scripturaire, c'est-à-dire la mise en représentation des lois d'un système spécifique qui consiste en la performance, dans un espace et un temps donnés, d'un élément signifiant en train de devenir visible, en train d'advenir à ce qu'il n'est pas encore. «Je décris ce qui est en train de se faire " ${ }^{3}$, dit Sarraute. Cet élément, quel est-il? D’abord et avant tout c'est le discours, parole en jeu, au sens où elle se donne à voir sur scène - scène de l'écriture -, où elle se manifeste comme substance vivante en train d'advenir comme spectacle. Mais c'est par ailleurs aussi le silence dans et de ce discours.

Dans l'œuvre de Nathalie Sarraute, le silence n'occupe en rien la marge scripturaire. Au contraire: il est le substrat même du drame que constitue le 
tropisme, cette sensation infinitésimale servant de matrice à la course du signifiant. Je propose donc de m'attarder à cette dialectique du silence et de la forme, en tant que le silence se fait signifiant princeps d'une scène de représentation où se joue l'avènement du mot comme rature du silence. Celui-ci, loin d'être absence de forme, vide formel, instaure la discontinuité dans la représentation, ouvre l'instant pour voir ce qu'il révèle de commencement/recommencement, c'est-à-dire de mouvement de recherche; il est ce mécanisme obligeant à chercher l'entame, à signifier l'infime interstice où le mot advient et résiste à une loi qui le borde et le déborde tout à la fois. Le silence ordonne ainsi la monstration du verbe comme performance, mais en tant qu'il défaille et qu'il perd la voix: c'est donc de l'énonciation comme acte d'être-là qu'il faudra aussi traiter, le silence constituant ici le point de butée mais aussi le point de relance du système signifiant.

Il s'agira dans le présent article de voir comment le silence se donne à l'écriture comme sa déchirure, voire son quasi-abandon - dans les deux sens du terme. Inobjectivable, formel mais dans sa négativité, énigmatique aux yeux du sujet-lecteur qui se voit projeté sur l'écran lisse d'une pure altérité; ce sujetlecteur est projeté hors corps: hors du corps de la lettre mais aussi hors de son propre corps de références signifiantes. Car chez Sarraute, le sens se construit dans l'inter-dit, dans l'inter-position du silence et de la voix, du silence et du geste scripturaire. Le silence est tracé circonvolutif, prenant inlassablement la mesure d'une forme, de formes en constante désintégration sur le plan de la signification. Le silence concourt donc à faire taire la parole au profit de la voix, qui, elle, laisse affleurer non la signification du mot mais sa signifiance. Le silence, ainsi, est la condition obligatoire à ce qu'un son devienne image, à ce qu'une voix devienne écriture.

\section{THÉÂTRALITÉS}

On pourrait parler chez Sarraute, en utilisant la typologie à quatre temps de Peter Brook - théâtres bourgeois, sacré, brut et vivant - d'un théâtre sacré, qui rend l'invisible visible ${ }^{4}$, donc qui présentifie, qui actualise par la parole l'infigurable sur lequel son discours repose. Car ce théâtre s'articule non pas autour d'une situation dramatique sur le plan de l'action scénique - l'intrigue est neutralisée pour mieux qu'émerge le dialogue -, mais bien autour du mouvement d'une parole dont l'activité peut être retracée comme écriture in actu. Monique Wittig, se faisant en quelque sorte le porte-parole de tous les critiques et commentateurs de l'œuvre de Sarraute, souligne que «l'usage de la parole est devenu le thème exclusif de ses livres " ${ }^{5}$. Ceci revient à dire que l'écriture de Nathalie Sarraute peut être entrevue comme pure expérimentation du langage, dont le silence bien sûr participe. Ainsi:

Son théâtre est envisagé en tant que théâtre expérimental, ce qui ne signifie nullement qu'elle utilise des moyens d'expression théâtraux spécifiques mais qu'elle expérimente presque uniquement avec le langage. De ce fait, Nathalie Sarraute poursuit dans ses drames le même but que dans ses textes en prose. 6

Ce but, il consiste à dire, à nommer, à formaliser ce qui du tropisme persiste à faire silence ${ }^{7}$ : «Je cherche à montrer en le développant, ce qui se passe en nous en quelques secondes" 8 . Ce qu'il y a à voir, à entendre est donc toujours déjà là, présent comme mouvement: le travail de l'écrivain consistera à en traduire les sinuosités, à en mettre à l'épreuve la théâtralité.

Ce qu'il y a à connaître du tropisme, c'est son trajet silencieux vers le mot. Le silence: pratique textuelle, usage de la parole, opérateur tropismique, gestus du signifiant? Oui, car qu'est-ce que le tropisme, sinon l'avènement-événement du signifiant de la sensation? Cette infraction du signifiant n'est possible que par l'intervention d'un silence qui règle le jeu. Jeu de place, «espace potentiel» dirait Winnicott, plus que jeu de rôle: espace où ça joue de la parole et où la parole n'est plus vaine.

En tant qu'espace structurel où se déploie une autre scène, le théâtre de Nathalie Sarraute pose ainsi la théâtralité comme son motif formel premier. Bien sûr, la conception aristotélicienne du théâtre nous le 
donne à penser comme une espèce de la poïesis, laquelle cherche à produire un effet par le truchement d'un objet, mais d'un objet qui engendre l'écriture dans la mesure où il génère une ouverture qu'investissent la parole et le travail de la forme. Déjà se dessine avec Aristote une dialectique de la présence et de l'absence, qu'on retrouve aussi chez Daniel Sibony: «Représenter, c'est rendre active une présence en la projetant sur un espace où elle est plus parlante ${ }^{9}$. Le silence est ici pointé, en ce qu'il parle plus que la parole même. N'est-ce pas là le propre du poétique que de faire «apparaître l'invisible» ${ }^{10}$, c'est-àdire montrer du doigt le lieu où l'interstice se fait appel de signes?

Theatron, «lieu d'où l'on regarde», et, pourrait-on ajouter, d'où la parole regarde ce silence qui s'agite à la source même des sensations ${ }^{11}$. L'enjeu, le jeu du théâtre ne consisterait-il pas ainsi à faire sortir la parole de sa suffocation, à rendre palpable sa théâtralité, c'est-à-dire sa nature d'espace silencieux? «Le théâtre est l'art de jouer avec la division en l'introduisant dans l'espace par le dialogue " ${ }^{12}$, disait Blanchot. Jeu de la coupure, de l'attente, du retrait momentané, pour que rien, justement, passe à l'écriture...

H. 2: Ah, vous savez, je dois vous dire... Il faut que je vous parle...

F.: Oui? De quoi?

H. 2: C'est idiot... c'est très difficile... Je ne sais pas comment... Par où commencer...

F.: Allezy toujours. Qu'est-ce que j'ai encore fait?

Oh rien. Rien. Rien justement, vous n'avez rien fait. Rien dit.

Vous vous taisiez...

F.: Il fallait que je parle?

H. 2: Oui, ça aurait mieux valu...

Que je parle quand? Que je parle de quoi? je ne comprends rien.

H. 2: Si, si... vous allez voir, vous allez comprendre... 13

Dans le théâtre de Sarraute, donc, il se passe rien, rien qu'on puisse enfermer dans une forme d'intrigue dramatique. Tout se passe comme si cette écriture se faisait le témoignage "d'un secret sans contenu, sans contenu séparable de son tracement performatif ${ }^{14}$.
C'est aussi là la part d'éthique de cette écriture, qui tend à présentifier, à figurabiliser, par l'usage d'un verbe apparemment simple mais extraordinairement chargé d'affects, ce qui a l'air d'être rien mais qui constitue une présence réelle, insistante, répétitive, qui ne cesse d'advenir dans l'énonciation. L'écriture de Sarraute, comme "parole d'écriture» dirait Blanchot ${ }^{15}$, cherche à inscrire le lieu où l'écriture a lieu, à faire se poser le regard là où ça vibre, où il y tension et appel.

Alors, Sarraute, dramaturge ou poète dramatique? "[...] le poétique, c'est ce qui parle dans le langage, de la part de l'Autre, dans le partage du dire» ${ }^{16}$. Une sorte de musique, état préliminaire à l'acte poétique, se ferait ainsi entendre, un certain ordre des choses, une disposition implicite et non articulée. L'écriture s'offre donc comme lien sonore et comme lieu du murmure soumis à l'appel du contact et de l'écoute. Ainsi, l'écriture de Sarraute est-elle une écriture interprétative qui court à corps perdu après son biendire et qui «se caractérise par la mise en jeu de la défaillance de la lettre, la mise en évidence du manque sur lequel échoue toute prise littérale» 17 .

[...] ça ne ressemble à rien, ça ne rappelle rien de jamais raconté par personne, de jamais imaginé... c'est ça sûrement dont on dit qu'il n'y a pas de mots pour le dire... il n'y a pas de mots ici... 18

Et pourtant, ce rien, si petit soit-il, Sarraute n'a de cesse de l'articuler, d'en soutirer le souffle, de le «dénoncer "; dans une sorte de passion répulsive à l'endroit de ce qui tient lieu d'origine et de fin à la fois, un silence se fait petit rien et donne lieu au lieu, engendrant la scène.

C'est précisément parce que ça ne ressemble à rien «de jamais raconté» que dire, écrire sans cesse devient légitime pour le sujet de l'écriture qui vise justement à surinvestir ce silence pour l'exténuer. Plus qu'une motivation à écrire, c'est une obligation, une injonction qui s'emballe en un mot: «Écris!». Ce qui peut aussi s'entendre: "Questionne!», avec toute la portée subversive que contient un tel mandat, lequel oblige à la suspicion à l'égard du mot, de l'écriture, de leurs conventions et inadéquations. 
Parce que rien a lieu, c'est du lieu que ce rien s'invente, se parle comme ce petit rien qui fait l'«objet» des conversations quotidiennes. De l'anonymat, ou du silence du rien, à ce petit rien qui semble, lui, pouvoir s'arrimer aux mots, ce passage même constitue le drame et sa structure théâtrale. Partant, rien ne peut exister que comme en train d'advenir, en pleine performance. S'attarder à ce petit rien de silence permet d'observer les rouages du passage à l'acte - le dialogue -, comme le ferait une loupe grossissant les moments de discontinuité dans le continuum dialogique en en repérant les motifs de répétition. Le temps du dialogue étant souvent chez Sarraute sous le signe de la confession, c'est donc nécessairement un temps lent, la lenteur pouvant être alors expérience concrète du ralentissement, ici vécu "physiquement», et qui permet d'entendre les pulsations du discours et de voir leur présence amplifiée. Un tempo est ainsi rendu visible, audible qui présentifie le temps de la naissance du tropisme et en fait une durée sensible, un repère dramatique: il répond donc à une nécessité réelle et logique en ce qu'il décrit l'ordre du surgissement, intégré au temps de l'écriture. Non pas analysés mais ressentis, ces mouvements dramatiques silencieux sont rendus par le rythme et la métaphore, puisqu'en eux-mêmes ils sont a-signifiants. Pour devenir signes, ces mouvements doivent être transmis par et dans le temps. Or, chez Sarraute, cette transmission se fait dans un «temps qui ne passe pas» ${ }^{19}$, mais qui crée l'espacement nécessaire à l'apparition de l'écriture et à l'expérience de la scène. Le temps de l'écriture étant celui d'un après-coup évolutif, celui d'une reconquête, d'une réélaboration langagière, voire sensitive, où s'étalerait le temps trop «impulsif», dirait Sarraute, de la sensation, la visée est donc d'inventer le lieu de silence d'où l'on écrit pour authentifier la sensation comme absence éminemment présente, et ce à tous les instants, non pour la communiquer et la décrire mais pour en témoigner. La sensation est toujours originaire, annonciatrice de la lettre à venir ; cependant, puisqu'elle est leurre - et revendiquée comme tel -, elle est aussi pur travail de l'imaginaire, entreprise menant à la nomination. Elle n'apparaît que par le jeu d'un regard en biais porté sur les mots; elle se donne à entrevoir dans des scories, donc des pleins en creux allégoriques. La sensation, le tropisme formant une tension, un surgissement, l'écriture théâtrale tentera du coup - profitant de l'espace scénique comme lieu de l'effraction, comme le dit Françoise Asso - d'en rendre compte dans son effectuation, dans son processus d'éruption et d'intrusion.

\section{OUVREZ...}

Comme à travers une fêlure dans une paroi lisse, une fine craquelure, quelque chose se glisse doucement... subrepticement cela s'insinue en lui, quelque chose d'informe, de gluant avec une obstination sournoise cherche en lui son chemin... 20

J'en reviens à Ouvrez, dont voici l'incipit:

Des mots, des êtres vivants parfaitement autonomes, sont les protagonistes de chacun de ces drames.

Dès que viennent des mots $d u$ dehors, une paroi est dressée. Seuls les mots capables de recevoir convenablement les visiteurs restent de ce côté. Tous les autres s'en vont et sont pour plus de sûreté enfermés derrière la paroi.

Mais la paroi est transparente et les exclus observent à travers elle.

Par moments, ce qu'ils voient leur donne envie d'intervenir, ils n'y tiennent plus, ils appellent... Ouvrez.

Des mots sont là, sur la scène de la représentation. Ils parlent entre eux, ils parlent les uns des autres dans un pur dialogue: ils sont donc en mouvement, en acte, portés par le silence du tropisme. Deux clans, deux espaces se distinguent et s'opposent: l'un et l'autre côté de la paroi, soit celui des mots recevables, bien disciplinés, puis celui des autres, qualifiés de chiens fous sur lesquels on ne peut compter, ces mots avec lesquels on ne sait jamais à quoi s'attendre puisque ce sont des mots revendicateurs, inquisiteurs. "Pour moi, les mots sont des choses vivantes", des entités agissantes, soutient Sarraute ${ }^{21}$. «Ils jouent, se répondent, se font écho. Ils se répercutent. Ils se reflètent, ils miroitent» 22 . Entre les deux groupes, un 
espace, un entre-deux, une barrière: le jeu du silence, c'est-à-dire cette paroi transparente où ça se joue.

- Oh, qu'est-ce que c'est?

- Mais oui, c'est à ne pas croire, c'est «Je vous aime»... c'est bien lui...

- Son bon vieux cour s'est ému, il veut aller de l'autre côté, au secours de ces mots qui courent à leur perte...

- Lui, "Je vous aime», ce pauvre vieux à la retraite depuis si

longtemps, il veut reprendre du service...

- "Je vous aime» qui ne se produisait plus que porté, soutenu par les voix d'acteurs bien entraînés dans des pièces $d u$ répertoire... Il veut maintenant tout seul, sans soutien... - C'est touchant, c'est pitoyable de voir comme il s'efforce de redresser ses vieux membres perclus, il s'avance péniblement sur ses jambes torses... vous entendez sa voix chevrotante... «Je vous aime», oui, c'est moi, je veux entrer... Ouvrez...

- Il arrache ses habits de théâtre, il exhibe son corps sénile...

- Avec ce "vous" qui le rend grotesque...

- Qu'on lui mette un "tu», et comme ça le rajeunirait... il serait tout à fait présentable... 23

"Je vous aime», ce sont des mots conventionnés, des mots tout juste bons à être donnés à dire par un acteur de répertoire. Des mots usés, galvaudés qui appartenaient autrefois au lieu commun mais qui en ont été exclus au profit d'un "Je t'aime» dorénavant beaucoup plus convenable. C'est ainsi qu'ils seront littéralement jugés lorsqu'ils tenteront de traverser la paroi pour aller rejoindre les autres mots, ceux dont on dit qu'ils sont doués pour faire le travail qu'on exige d'eux, ceux qui représentent adéquatement et sans le trahir celui qui s'en sert. Passer la paroi, abolir pour un temps le silence ... et croire à cette petite réussite.

Ouvrir. Comme Cratyle lève le doigt, se taisant, pour faire le geste d'écrire en se taisant. Écrire. Peu importe quoi. Seul le passage à l'acte compte pour l'instant. Ouvrez. Ce texte, je le reçois ici comme un art poétique: la poursuite d'un dire, selon une configuration discursive singulière, répondant à des modalités scripturaires différentes de celles de la fiction mais qui serait elle aussi partie prenante de l'ordre de l'écriture fictionnelle, un peu comme l'est le commentaire chez Blanchot. Une insatisfaction fondamentale vis-à-vis une parole instable mais stationnaire, approximative mais étouffante, constamment relancée, questionnée, rouverte. Une approche, un affleurement de l'écriture, puis une dessaisie devant l'impossibilité de toute réponse fixe. C'est là le travail fondateur du tropisme, du mouvement intérieur, du silence.

Et pourtant, l'écriture ne se tait pas... Forcenée, elle s'acharne à supporter les insuffisances du langage, ses fractures, ses ratés, hantée qu'elle est d'avoir le dernier mot. Elle produit, et s'ouvre à ce vide qui la fonde, laissant entendre "[...] un assourdissant silence par-dessus le vacarme [...]»24, comme le souligne JeanYves Tadié en ouverture de son introduction aux Euvres complètes de Sarraute. Un assourdissant silence, une paroi vibrante et transparente.

\section{LE SILENCE À L'GEUVRE}

Ouvrez, scène vingt-trois:

- Vous avez l'air mal à l'aise... Qu'est-ce qui vous est arrivé?

- Vous qui étiez tout le temps là, vous avez dû vous apercevoir de quelque chose?

- Mais non, vous nous avez vus... on a répondu aussitôt à toutes les convocations...

- C'était facile, c'était la plus banale des conversations. Du tout-venant... Impossible de faire un faux-pas...

- Dès que le répondeur a été décroché, nous sommes venus saluer comme il se doit quelqu'un de sympathique, de gentil... - Mais vous qui receviez ce qui arrivait de l'autre bout du fil, vous savez bien ce qui vous a mis dans cet état... Alors dites-le... - On se sent mal...

- Mais pourquoi? Faites un effort...

- Eh bien, quelque chose est arrivé à «C'est»...

- $\dot{A}$ «C'est»?

- Oui, "C'est» a été malmené devant nous, on lui a enlevé son «t»...

- Et il a fallu l'accepter sans rien faire.

- Enlevé son «t»? Comment?

- Dès que l'écouteur a été décroché est venu de là-bas, précédant "Antonin», un «C'es» amputé de son «t»... C'es... Antonin... - Une crevasse s'était ouverte... un vide, tout à coup, qu'il fallait enjamber... 
- Ce «t» qui est une de nos fiertés... Quelle autre langue le possède?

- Cette grâce qu'il a, cette légèreté.

- Cette courtoisie... dès qu'un vide s'ouvre, difficile à franchir,

«t» s'élance de c'es... comme une passerelle légère...

- Oui, un «t» qui viendra délicatement se poser de l'autre côté...

- Oh, si délicatement... en effleurant à peine...

- Ah mais c'est encore trop. L'autre que «t» touche si

légèrement, le sent posé sur lui, adhérant à lui, insupportable...

- Il lui semble que ce «t» collé à lui le rend grotesque...

"Tantonin»... voilà ce que ce «t» en a fait... Tantonin... «Cé

Tantonin »...

- Il n'en veut à aucun prix...

- Ce «t» doit être détruit... 25

Une conversation téléphonique ordinaire, banale. Et une lettre. Un petit «t», en rien inoffensif, offensant bien au contraire. Une faute, un faux pas, à lire littéralement: «La lettre, ça se lit. Ça semble même être fait dans le prolongement du mot... Ça se lit et littéralement» ${ }^{26}$, disait Lacan. On voit bien ici ce qui est fait de ce "prolongement du mot»: déporté, délié, passant d'un son à l'autre, par-delà le mur du silence; le «t» qui au départ appartenait à un groupe, "C'est», se fait transfuge et passe au nom "Antonin", créant du coup un monstre. Voilà maintenant "Tantonin", ou encore "C'es... Antonin", double défiguration du signifiant. " $T$ » est passé ailleurs, de l'autre côté de la paroi, pour instaurer une sorte d'unité sonore nouvelle, abolissant le silence qui auparavant authentifiait, fixait le signifiant dans un lieu circonscrit. Et pourquoi? Parce que ce silence, on y croit puisqu'on le voit, on le voit à l'œuvre, on l'entend parler, ou plutôt faire acte de parole, on en constate la bouleversante portée, de la même façon $\mathrm{qu}^{\prime}$ " [...] une parole ne devient une parole que dans la mesure exacte où quelqu'un y croit» 27 .

Quelque chose palpite dorénavant, silencieusement, entre "C'es» et «Antonin». Un entredeux instauré comme par mégarde, par inadvertance. Un mouvement du dire crée ainsi une distance avec le dit, installant un silence suspendu comme signifiant central autour duquel la scène du drame se jouera. " $T$ " n'était pas demandé: il est venu, tel un aveu, à l'autre qui le reçoit comme un intrus indélicat. L'inquisition commence: on va, ce petit "t», l'ausculter infiniment et tenter de comprendre comment le silence qu'il a drainé avec lui, et qui à présent génère toute l'action dramatique, a pu réussir à bousculer l'ordre d'un langage autrefois totalitaire, si policé, si ordonné.

Le centre de gravité du drame passe ainsi de la parole au silence, comme c'est le cas dans tout le théâtre de cette auteure, puisque c'est la parole qui sert le silence, le transporte dans un réel face à face, comme si deux espaces discontinus, l'un logorrhéique, l'autre minimal, se toisaient. Partant, ce silence, loin d'être un vide énonciatif, est tenseur de l'énonciation. La seule parole effective dans l'instant présent et prégnant de la scène est celle qui se dit en silence, en tant que le silence est un signe sensible, non un signe intelligible - bien que ses retombées le soient. Là où le mot stagne, le silence ébranle l'ordre des choses et mobilise des forces dramatiques inattendues. Dorénavant, c'est ce qui, du mot, se tait activement qui autorise la scène; le mot qui parle, croyant ainsi agir, est agi et court le risque d'être englouti par sa parole. Pour éviter cela, la stratégie est sinon simple du moins efficace: emprunter la forme du silence pour s'éviter d'être confiné dans le rôle ingrat de spectateur du silence. «Être là en tant que ça se tait» ${ }^{28}$, voilà l'enjeu, voilà l'issue. Du silence subi faire un silence imposé, riposter en s'appropriant la posture de l'autre, habiter le silence de l'autre et, ultimement, le retourner à son propre silence, nécessairement hostile et vide.

L'écriture de Sarraute fait donc du silence un «régime d'énonciation littéraire particulier ${ }^{29}$ qui tend à stupéfier le dialogue. Car le silence, toujours, surprend la parole, la prend et la déporte ailleurs. C'est là que le paradoxe s'installe: qu'est-ce qui, du tropisme ou du mot, autorise l'autre? qu'est-ce qui, du silence ou de la parole, porte l'autre? Aporétique, ce problème n'en demeure pas moins central dans toute l'œuvre de Sarraute. C'est toute la question du système de la représentation en tant que tel qui est par cela convoquée. 


\section{DU LIEU DU SILENCE}

Parlant de sa première tentative d'écriture théâtrale, Sarraute écrit:

L'idée m'est venue, quelque temps après, sans que je sache bien ce qui pourrait en sortir, d'un certain silence. Un de ces silences dont on dit qu'ils sont "pesants». Je mentirais si je vous rapportais comment de ce silence un texte a jailli, comme le ruban du chapeau du prestidigitateur ou, pour employer une comparaison plus modeste, comme le ruban de la fente d'un téléscripteur. [...]

Toujours est-il que tiré par ce silence un dialogue a surgi, suscité, excité par ce silence. Ça s'est mis à parler, à s'agiter, à se démener, à se débattre... [...]. 30

Le silence, on l'a vu, instaure le mouvement de recherche tropismique. "C'est la performance du silence qui fonde la compétence du dialogue. Celui-ci tire ainsi sa force du manque même sur lequel il repose» ${ }^{31}$. Le silence organise donc la mise à l'épreuve de la parole en l'attirant vers lui pour la contaminer, tel un troisième terme qui infiltrerait la relation dialogique pour présentifier l'écart, réinventer la relation duelle entre interlocuteurs, et finalement paralyser le dialogue. Dorénavant, la parole est issue du silence et vouée au silence, lequel a pour mandat de la faire résonner.

«Les paroles, dit Sarraute dans L'Ère du soupçon, possèdent les qualités nécessaires pour capter, protéger et porter au-dehors ces mouvements souterrains à la fois impatients et craintifs» ${ }^{32}$. Le tropisme sans ce à quoi il donne forme n'existe pas. Capter, protéger et porter audehors, seul le signifiant peut exécuter cette tâche qui lui donnera chair et le présentifiera aux yeux de l'autre, lecteur ou "personnage». Un point, donc, d'ordre langagier, qui sert de matrice, d'où toute l'activité de parole irradie et auquel tout finalement aboutit, après que le dialogue ait terminé d'achever sa proie.

À peine cette chose informe, toute tremblante et flageolante, cherche-telle à se montrer au jour qu'aussitôt ce langage si puissant et si bien armé, qui se tient toujours prêt à intervenir pour rétablir l'ordre - son ordre-saute sur elle et l'écrase. 33
Ainsi, tout part d'une fêlure superficielle chez l'un (un mot, d'un côté de la paroi), fêlure du silence accompagnée d'une sensation souterraine mais persistante chez l'autre (un autre mot, de l'autre côté), lequel cède alors à ces mouvements intérieurs dont la part d'infigurable l'envahit et qui cherche à rendre audibles ces tropismes silencieux qui l'habitent comme de véritables hantises.

J'avais toujours pensé qu'il ne me serait pas possible d'écrire pour le théâtre parce que tout y est dit dans le dialogue et que chez moi ce qui était important c'était ce que l'on appelait la «sousconversation", le "pré-dialogue». Le dialogue, c'est l'affleurement en dehors de ce qui a été préparé par ces mouvements intérieurs, ces tropismes. Il me semblait impossible d'écrire tout cela en dialogue. Puis un jour, quand on m'a demandé une pièce pour la radio de Stuttgart, je me suis dit que peut-être il serait amusant, intéressant de mettre dans le dialogue de théâtre justement tout ce qui est pré-dialogue dans le roman. 34

On peut donc dire que la trame de ces microdrames sarrautiens est faite de paroles produites à partir de tropismes, de petites «inquiétantes étrangetés» stimulantes, sensations qui laissent entendre un lieu silencieux où quelque chose serait toujours déjà inscrit. Les tropismes de l'un sont ainsi générés par les tropismes de l'autre, auxquels ils répondent en écho par l'invention du dialogue. Il s'agit ce faisant d'aménager, de dégager un espace où, dans la parole, la sensation ressentie au contact de l'étranger pourra poindre et ainsi générer un semblant d'échange, soutiré au silence. Toutefois, cette sensation ne peut jaillir que comme mi-dire, donc comme mal entendue dans la parole. Ce que l'un des pôles dialogiques percevra de l'énonciation de la sensation chez l'autre sera donc partiel, reconduisant ainsi de façon infinie le «mal-entendu». Il ne peut par conséquent jamais y avoir d'authentique rapport dialogique, parce que le dialogue est un rendez-vous manqué (quoiqu'il envahisse tout l'espace textuel jusqu'à se substituer au récit), entaché, enveloppé de silences qui, dans un registre qui leur est propre, disent eux aussi la sensation tropismique en forçant les signifiants à dire. 
La parole de ce dialogue, parole pleine en ce qu'elle «se définit par son identité à ce dont elle parle» ${ }^{35}$, arrive donc à transmettre une part d'innommé en tournant autour de la sensation comme un rapace autour de sa livre de chair, parole "qui fait acte» ${ }^{36}$ sur le mode de l'évocation - ce qui nous éloigne passablement de la représentation formelle de l'objet sémiotique. Et il s'agit bien ici de l'innommé, car la sous-conversation ne correspond pas au non-dit, mais à l'implicite où le silence est «une fonction ancillaire au dire " 37 , une épaisseur de la sousconversation, qui se manifeste notamment dans l'usage surabondant des points de suspension, au cœur desquels le silence se donne à lire comme lieu d'énonciation privilégié du tropisme. Ainsi:

[...] les points de suspension, en particulier, n'ont pas pour fonction de "suggérer un indicible», mais de lier entre elles, d'une liaison paradoxale puisqu' elle se fait dans un blanc du langage, les images et notations fragmentaires que fait naitre la sensation. 38

Par conséquent, le dire mis en partage dans le dialogue témoigne qu'il y a du secret, du (presque) rien silencieux, mais d'un silence qui ne relève pas d'une intériorité privée qu'il faudrait dévoiler. Le silence est au contraire central et partageable - c'est même une obligation que de le disséquer sur la place publique. Pourtant, ce silence reste irréductible à sa nomination puisqu'il a pour effet immédiat d'altérer le signe: c'est précisément pourquoi il est entendu comme cette souffrance qui circule sur la scène de l'écriture, dans la forme même du dialogue; il est une langue autre dont on offre en quelque sorte la «traduction» formelle.

- Et voici le moment où, pour de bon, la paroi s'abaisse et ceux qui étaient derrière... qui n'avaient jamais bougé de là, qui avaient tout observé, arrivent...

- Vous avez bien regardé? Que s'est-il donc passé...

- Oh, rien de grave, juste un moment d'absence... un léger

assoupissement.

- Ça s'est vu?

- Non... sûrement pas...
- Même pendant cette somnolence, des mots sont venus comme il le fallait... 39

«[...] rien de grave, juste un moment d'absence... un léger assoupissement»... Une chaleur ambiante, un grand vide, un suspens où les mots viennent passer, puisqu'il le faut bien. C'est donc d'une lutte effrénée contre la part d'informe du silence qu'il est question, lutte scripturaire qui inscrit un corps signifiant là où est entrevue une dissolution langagière. Inscrivant le signifiant dans sa course, elle donne lieu à la fiction et répond à l'insoutenable du silence par la mise en pièces de la parole.

Or, cette parole dialogique, dans ce qu'on appelle le logodrame sarrautien, ne suffit plus, cela est très clair. Elle ne suffit plus à sa tâche pour la raison simple qu'elle se voit attribuer un double emploi: dire le silence et le faire taire du même coup. Le tropisme silencieux rendu présent dans et par cette parole constituerait donc un état de stress devant un ressenti qui (se) parle par un taire, et partant une dynamique scripturaire, puisque ce taire-là est venu se glisser dans la parole comme trace d'une énonciation dont on ne saurait rien - ou dont on saurait le rien qu'elle transporte.

C'est une image et puis une autre... ce sont des bribes de conversation, ou bien juste une intonation, un accent qu'un mouvement rapide traverse, qui sont comme parcourus, secoués par une brève convulsion. 40

Et cette convulsion, cette lettre de silence, il faut la rechercher, la susciter, puis la capturer, l'attacher puis l'isoler pour la dépecer, l'interpréter. Convulsion du silence, vibration du silence, chair érectile se devant d'être vue, entendue, alors qu'elle passe dans le signifiant:

Il est impossible qu'ils ne le voient pas. C'est là, surgi du néant.

Cela se dresse, se déploie avec assurance, avec une audace tranquille.

Au centre de cela il y a quelque chose d'indestructible. Un noyau qu'il n'est pas possible de désintégrer, vers lequel toutes les particules convergent, autour duquel elles gravitent à une vitesse si énorme qu'elle donne à l'ensemble l'apparence de l'immobilité. 
Autour de cela des ondes se répandent, tout oscille, tout vibre autour, si on s'en approche on se met à vibrer. 41

En somme, le travail de l'écrivain consiste à abolir tout en l'instaurant l'interstice de silence, à faire s'interpénétrer le langage et la sensation, puisque les tropismes, partageant ici l'arbitraire du signe saussurien, ne portent au départ aucun nom, n'appellent aucune forme de façon immédiate. Le sens est donc un espace vide, un blanc à remplir, mais à remplir de signifiants neufs; ainsi, la signification n'est qu'une répercussion du flux tropismique. Et cette signification naît de cette incontournable obligation d'établir le contact avec un autre signifiant, donc de passer par le silence - ou pardelà la paroi qui le présentifie.

Ce qui, du tropisme, est premier, est le mécanisme de défense contre le silence qu'il implante, avant même d'offrir les conditions formelles de son énonciation. Ce qui en résulte est un cri, que le "personnage» se tue à vouloir organiser pour se le rendre signifiant, pour le faire passer d'un magma informe et silencieux à un signifiant palpable pouvant être assimilé dans et par le discours. Ce trajet, qui est celui de l'écart entre le signifiant et le tropisme, fera ainsi passer un substrat langagier a-signifiant à une expérience de la signifiance même. Reste le trajet... car "cette fusion du langage et de la sensation intacte crée quelque chose de particulier, qui a une existence propre; quelque chose qui procure une jouissance d'ordre esthétique " ${ }^{2}$. Cela permet d'ouvrir du temps, celui de la scène, pour dire, justement, ce qui du sens s'absente dans l'impossible cohérence entre les formes. Évacuant le sens, c'est la signifiance qui s'installe; alors la parole se produit et «le texte répète ce dont il parle, en dépit de variations continues dans la manière de dire " 43 . Ce temps vide de l'origine se traduit par conséquent par une narration du désert, puisque ce n'est pas ça.

\section{NOMMER CE SILENCE}

Donner forme à la présence silencieuse: la mettre en forme dans le littoral de la parole dialogique. Car la menace, l'angoisse, la souffrance, c'est bien là qu'elles se trouvent, dans ce qui revient dans l'énonciation comme un déjà «senti» qui insiste. Et puisqu'on l'a senti, réellement, pourquoi ne pas en inventer la scène, pour en faire taire le mal? Pourquoi ne pas donner libre cours au processus créateur jusqu'à en faire un motif dramatique? Puisqu'on ne peut non plus se soustraire à la portée représentative du silence, puisqu'il rend palpable l'indicible de la relation du sujet au sens, le silence s'en trouve donc régisseur de la mise en dialogue. Il est l'épreuve du dire, constituant en somme la seule vérité: celle qui ne peut accéder à l'existence qu'en se répétant sous des formes plurielles constamment reconduites dans un jeu de miroir malsain et jouissif. Si un "personnage», un mot dit quelque parole qui, dans l'énonciation, tend à entrouvrir le lieu de cette vérité, l'autre inévitablement tentera de s'approprier ce lieu. C'est un geste d'agression, rien de moins. S'ensuivra une mécanique de la reprise et de la variation qui, parfois, en vient même à prendre les allures d'un combat meurtrier sans fin ni but autre que celui de lutter, en pure perte. Lutter pour la survie de sa voix s'entend, parce qu'il s'agit bien ici d'un combat à la vie à la mort, d'un incessant processus de déchirement, de décloisonnement du silence.

Ainsi, ce que construit Sarraute, c'est un système esthétique de la possibilité, d'où le privilège accordé à la brièveté de l'énonciation, entrecoupée de suspens, qui fait que, en bout de course, cela ne prend pas: «Un double désir agit celui qui écrit: trouver la formule qui résume, qui condense son objet stupéfié, mais qui échappe pourtant dans cette même énonciation à son dit» 44 . Il s'agit donc d'une éthique du refus de la formule galvaudée, de l'assertion définitive, de l'impossibilité d'un verbe totalisateur qui serait mort du désir d'écriture. Tout cela au profit des inlassables détours de la scène d'écriture du Livre.

Bien sûr, il n'y a jamais de véritable mort du drame chez Sarraute, parce qu'il n'existe pas de lieu sans acte discursif, de silence de mort où la demande de dialogue serait évitée. Seulement, il y a ces petites morts de la parole où le silence semble lui indiquer qu'elle n'aura jamais le dernier mot. La parole ne fait que traverser 
furtivement les lieux, les scènes du silence, ne laissant derrière elle qu'un rayonnement signifiant, qu'une errance privée de lieu où elle pourrait enfin se figer... et mourir. Plutôt que de mourir au silence, mieux vaut pactiser...

Somme toute, donner forme au dire, ouvrir le dialogue, c'est d'abord s'essayer à nommer le silence, tenter d'en localiser la lettre. C'est pourquoi il me semble qu'une sémiotique du silence devrait inévitablement se confronter à le considérer d'un point de vue topologique, puisque c'est d'abord comme lieu scripturaire que le silence agit et crée l'agir. Le silence est agent et non objet discursif: ne s'en écrivent que les retombées, les effets, c'est-à-dire du semblant qui passe nécessairement par l'écriture pour être signifié comme présence signifiante. Sarraute, dans cet ordre d'idée, forge l'écriture d'un lieu qui est le silence, comme le fait Lacan en écrivant $a$ dans ses mathèmes: il le place en un lieu, mais il ne le nomme pas. Pourquoi? Parce que ça n'a pas lieu d'être autrement.

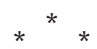

Revenons, pour conclure, à Ouvrez. Ce «t» qui s'écrit, qui tombe dans le dialogue et dont les motivations sont incompréhensibles aux yeux des autres mots, il faut le faire taire, c'est-à-dire tenter de le parler, de l'écrire. Le faire taire pour ne plus qu'il fasse silence, pour stopper sa course signifiante. Tacere. Silence actif, aboutissement et commencement du drame, signant le retrait de la parole mais aussi son «invention». La parole ne serait-elle que ce «support» formel servant à "authentiquer le silence» 45 ? Car on ne peut que scander le silence, prendre la mesure des points d'arrêt qu'il présentifie, en mesurer l'intensité, la portée vibratoire.

L'écriture de Sarraute est sous le signe de l'absence de toute autorité sémiotique efficiente. La promesse de catharsis n'y est d'ailleurs jamais réellement fondée, assise sur autre chose que des ruines circulaires dont aucune parole, aussi obsédée soit-elle par sa propre délivrance, n'arrive à s'extirper. Son épreuve du silence demeure donc son horizon, sa fiction.

Écrire ne peut aller sans se taire; écrire, c'est, d'une certaine façon, se faire "silencieux comme un mort", devenir l'homme à qui est refusée la dernière réplique; écrire, c'est offrir dès le premier moment cette dernière réplique à l'autre. 46

Montrer ce qu'on ne saurait voir, faire entendre ce qui ne saurait l'être: n'est-ce pas là la plus simple façon de se figurer le tropisme? Car l'éthique de la parole, la parole éthique, est une mise en mouvement du dire contre le déjà dit: or, le silence ne saurait être un déjà dit. Voilà pourquoi l'éthique, comme le silence, est brisure, cassure, fissure, éclatement du mot en ses lettres - puisque l'éthique et le silence exigent qu'on lise à la lettre -, soulignant parfois que le rapport entre voyelles et consonnes, comme le rapport d'amour à l'autre, n'a rien d'une évidence et qu'il faut y reconnaître le jeu du désir.

L'aspect ludique, ironique, voire sarcastique, de l'écriture de Nathalie Sarraute se donne donc à entendre dans l'amplification de la présence du silence dans des mots et des gestes, dans l'effraction devenue excessive d'une légère oscillation tonale par exemple, qui gonfle par la suite et devient un monstre d'envahissement. Ce qui est proprement comique dans cette théâtralité scripturaire, et tragique à la fois, c'est le décalage pourtant microscopique mais infiniment exagéré qui existe entre la représentation qui est instaurée à partir et autour du signifiant et le signifiant lui-même.

Sarraute chercherait-elle, livre après livre, «un audelà du langage dans le langage même» 47 ? Une sorte de "parole brute»?

Silencieuse, donc, parce que nulle, pure absence de mots, pur échange où rien ne s'échange, où il n'y a rien de réel que le mouvement d'échange, qui n'est rien. Mais il en va de même pour la parole confiée à la recherche du poète, ce langage dont toute la force est de n'être pas, toute la gloire d'évoquer, en sa propre absence, l'absence de tout: langage de l'irréel, fictif et qui nous livre à la fiction, il vient du silence et il retourne au silence. 48 
"Le silence est le fond du monde et son chaos", disait fort justement Pierre Fédida dans «La Table d'écriture " 49 . En tant qu'il s'efface et s'ouvre à sa mort, puisque le silence se constitue toujours déjà de ce double mouvement d'ouverture et de fermeture, force est d'écrire ce dont on ne peut parler. En cela, inaugural et préfiguratif, le silence est œuvre à venir, mais en sa propre tessiture...

\section{N O TES}

1. N. Sarraute, Tropismes, dans Euvres complètes, 1996, p. 8.

2. D. Rabaté, 1999: 116.

3. N. Sarraute dans un entretien avec I. Sadowska-Guillon, 1986: 16. 4. "J'aurais pu l'appeler le théâtre de l'invisible-rendu-visible». P. Brook, $1977: 65$.

5. M. Wittig, 1984: 70.

6. W. Floeck, 1992: 296. Je souligne.

7. Définissant le tropisme, Sarraute dit: «Ce sont des mouvements indéfinissables, qui glissent très rapidement aux limites de notre conscience; ils sont à l'origine de nos gestes, de nos paroles, des sentiments que nous manifestons, que nous croyons éprouver et qu'il est possible de définir. [...] Leur déploiement constitue de véritables drames qui se dissimulent derrière les conversations les plus banales, les gestes les plus quotidiens. Ils débouchent à tout moment sur ces apparences qui à la fois les masquent et les révèlent». "Préface " à L'Ėre du soupçon, dans Euvres complètes, p. 1553-1554.

8. N. Sarraute, dans Fauchereau et Ristat, 1984: 10.

9. D. Sibony, $1997: 148$.

10. N. Sarraute, "La Littérature, aujourd'hui ", dans Euvres complètes, p. 1662.

11. «Pour moi, le roman se rapproche, essaye de se rapprocher de la poésie; il tend, comme la poésie, à saisir au plus près de leur source, des sensations, quelque chose de ressenti. Les romans devraient devenir de grands poèmes ». N. Sarraute, dans Fauchereau et Ristat, 1984: 18. 12. M. Blanchot, 1969: 528.

13. N. Sarraute, Elle est là, dans Euvres complètes, p. 1475-1476.

14. J. Derrida, 1993 : 56.

15. Sous-titre du $1{ }^{\mathrm{er}}$ chapitre de L'Entretien infini.

16. D. Sibony, 1985: 60.

17. C'est ainsi que S. Leclaire définit l'interprétation psychanalytique dans Démasquer le réel, 1971: 25. Précisons qu'il considère cette mise en évidence du manque comme violente, «inacceptable et scandaleuse » en ce qu'elle a trait à la chose inter-dite, ce qui fait écho au théâtre sarrautien.

18. N. Sarraute, L'Usage de la parole, dans Euvres complètes, p. 924. 19. J.-B. Pontalis, 1997.

20. N. Sarraute, Entre la vie et la mort, dans Euvres complètes, p. 658.

21. Dans V. Forrester, 1983: 19.

22. N. Sarraute, Entre la vie et la mort, dans Euvres complètes, p.663.

23. Idem, Ouvrez, p. 110-111.

24. Dans N. Sarraute, Euvres complètes, p. IX.

25. N. Sarraute, Ouvrez, p. 63-64.

26. J. Lacan, 1975b: 29.

27. Idem, 1975a : 264.

28. F. Fonteneau, 1999: 138

29. D. Rabaté, 1999: 41.

30. N. Sarraute, "Le Gant retourné», dans Euvres complètes, p. 1708.

31. A. Rykner, 1996: 303.

32. N. Sarraute, L'Ėre du soupçon, dans Euvres complètes, p. 1597. Je souligne.

33. Idem, "Ce que je cherche à faire", dans Euvres complètes, p. 1704.

34. N. Sarraute dans un entretien avec I. Sadowska-Guillon, 1986: 14.

35. J. Lacan, 1966: 381.

36. J. Lacan, 1975a: 125-126.

37. E. P. Orlandi, $1993: 227$.

38. F. Asso, 1995: 48.

39. N. Sarraute, Ouvrez, p. 59.

40. Idem, Entre la vie et la mort, dans Euvres complètes, p. 666. 
41. Ibid., p. 677-678.

42. N. Sarraute, "Le Langage dans l'art du roman", dans CEuvres complètes, p. 1691.

43. M. Riffaterre, 1979: 76

44. D. Rabaté, 1999: 52.

45. S. Mallarmé, $1945: 387$

46. R. Barthes, «Préface» aux Essais critiques, 1993: 1169.

47. D. Rabaté, 1999: 23.

48. M. Blanchot, 1955: 38. Je souligne.

49. P. Fédida, $1976: 90$

\section{RÉFÉREN CES BIBLIO G RAPH IQ U ES}

Asso, F. [1995]: Nathalie Sarraute. Une Écriture de l'effraction, Paris, P.U.F.

BADIOU, A. [1992]: Conditions, Paris, Seuil.

BARTHES, R. [1993]: Essais critiques, dans CEuvres complètes, tome I,

Paris, Seuil.

Blanchot, M. [1955]: L'Espace littéraire, Paris, Gallimard;

[1969]: L'Entretien infini, Paris, Gallimard;

[1980]: L'Écriture du désastre, Paris, Gallimard.

Brook, P. [1977]: L'Espace vide. Pour une poétique de l'énonciation, Paris, Seuil.

DERRIDA, J. [1993]: Passions, Paris, Galilée.

FAUCHEREAU, S. et J. RISTAT [1984]: «Conversation avec Nathalie Sarraute", Digraphe, no 32.

FÉDIDA, P. [1976] : «La Table d'écriture», Nouvelle Revue de psychanalyse, $\mathrm{n}^{\circ} 16$.

FLOECK, W. [1992\}: «Nathalie Sarraute, dramaturge, à la recherche des pulsions intérieures de l'âme", Revue d'histoire du théâtre, n 44.

FOnTENEAU, F. [1999] : L'Éthique du silence. Wittgenstein et Lacan, Paris, Seuil, coll. "L'Ordre philosophique».

ForRester, V. [1983] : «Portrait de Nathalie», Magazine littéraire, no 196.
LACAN, J. [1966] : Écrits, Paris, Seuil;

[1975a]: Séminaire I. Les Écrits techniques de Freud, Paris, Seuil;

[1975b] : Séminaire XX. Encore, Paris, Seuil.

LALA, M.-C. [1999]: "À la pointe du style», dans Le Silence. La force du vide, s. la dir. de C. Danziger, Paris, Autrement, coll. "Mutations ", $\mathrm{n}^{\circ} 185$.

LECLAIRE, S. [1971] : Démasquer le réel. Un essai sur l'objet en psychanalyse, Paris, Seuil.

MALlARMÉ, S. [1945]: «Quant au Livre», dans Euvres complètes, Paris, Gallimard, coll. "Bibliothèque de la Pléiade».

ORLANDI, E. P. [1993] : "Silence, sujet, histoire», dans L'Esprit de société.

Vers une anthropologie sociale du sens, Bruxelles, Mardaga, coll.

"Philosophie et langage".

Ouellet, P. [1999] : «Pensées en cendres: penser ensemble», Spirale, $\mathrm{n}^{\circ} 169$.

PAVIS, P. [1985]: Voix et images de la scène. Pour une sémiologie de la réception, Lille, Presses universitaires de Lille.

PONTALIs, J.-B. [1997]: Ce Temps qui ne passe pas suivi de Le

Compartiment de chemin de fer, Paris, Gallimard.

RABATÉ, D. [1999] : Poétiques de la voix, Paris, José Corti.

RifFAterre, M. [1979]: La Production du texte, Paris, Seuil.

RYKNER, A. [1996]: L'Envers du théâtre. Dramaturgie du silence de l'âge

classique à Maeterlinck, Paris, José Corti.

SADOWSKA-GUILLON, I. [1986]: "Conversation avec Nathalie Sarraute.

À la recherche du temps présent", Acteurs, no34.

SARraute, N. [1996]: CEuvres complètes, Paris, Gallimard, coll.

"Bibliothèque de la Pléiade";

[1997]: Ouvrez, Paris, Gallimard, coll. «Blanche».

SiBONY, D. [1985] : Jouissances du dire, Paris, Grasset;

[1997]: Le Jeu et la Passe. Identité et Théâtre, Paris, Seuil.

VASSE, D. [1988]: La Chair envisagée. La génération symbolique, Paris, Seuil;

[1995]: Inceste et Jalousie. La question de l'homme, Paris, Seuil.

WitTig, M. [1984]: «Le Lieu de l'action", Digraphe, nº 32. 